\title{
Staphylococcus aureus induces autophagy in bovine mammary epithelial cells and the formation of autophagosomes facilitates intracellular replication of Staph. aureus
}

\author{
Heng Wang, ${ }^{1,2 *}$ Yuqi Zhou, ${ }^{1,2 *}$ Qicheng Zhu, ${ }^{1,2}$ Haozhe Zang, ${ }^{1,2}$ Juan Cai, ${ }^{1,2}$ Jianqiang Wang, ${ }^{1,2}$ Luying Cui,, \\ Xia Meng, ${ }^{1,2}$ Guoqiang Zhu, ${ }^{1,2}$ and Jianji $\mathrm{Li}^{1,2} \dagger$ \\ ${ }^{1}$ College of Veterinary Medicine, Yangzhou University, Yangzhou, Jiangsu 225009, China \\ ${ }^{2}$ Jiangsu Co-innovation Center for Prevention and Control of Important Animal Infectious Diseases and Zoonoses, Yangzhou, Jiangsu 225009, \\ China
}

\section{ABSTRACT}

Staphylococcus aureus is an important pathogen causing chronic and subclinical mastitis of cows. Autophagy is an important regulatory mechanism that participates in the elimination of invading pathogenic organisms. Here, we hypothesize that autophagy is involved in the process of Staph. aureus survival in bovine mammary epithelial cells (BMEC). In this study, we detected the expression of autophagy-related proteins during infection and assessed the effect of autophagosome formation and degradation on the proliferation of intracellular Staph. aureus. Infection with Staph. aureus increased the protein expression of microtubule-associated protein 1 light chain 3-II (MAP1LC3, also called LC3-II) and sequestosome-1 (SQSTM1, also called p62) in BMEC. After infection, the formation of the autophagosomes increased but the autophagosomes and lysosomes could not fuse normally to form autolysosomes. When the formation of the autophagosomes was enhanced or the degradation of the autolysosomes was inhibited, the number of Staph. aureus in the BMEC increased. However, the intracellular proliferation of Staph. aureus was slowed when formation of autophagosomes was inhibited. Therefore, autophagy was induced in BMEC challenged by Staph. aureus but the autophagic flux was obstructed. Inhibiting the formation of autophagosomes in BMEC facilitated the clearance of intracellular Staph. aureus, which may offer a new strategy for the treatment of mastitis in cows.

Key words: Staphylococcus aureus, bovine mammary epithelial cells, autophagy flux, proliferation

Received February 1, 2019.

Accepted May 1, 2019.

*These authors contributed equally to this work.

†Corresponding author: yzjjli@163.com

\section{INTRODUCTION}

Mastitis is an important disease in the dairy industry and is considered the most costly disease among dairy cattle, causing an economic burden in the dairy industry worldwide (Halasa et al., 2007). The decline in milk production and quality accounts for approximately $70 \%$ of the total cost of mastitis (Zhao and Lacasse, 2008). Subclinical mastitis is the main form of mastitis, with an incidence of 20 to 50\% (Pitkälä et al., 2004; Joshi and Gokhale, 2006; Olivares-Pérez et al., 2015).

Mastitis is usually caused by a microbial infection and Staphylococcus aureus is an important pathogen (Watts, 1988; Hillerton and Berry, 2005). Due to the resistance of Staph. aureus and its ability to evade the innate and adaptive immune responses, Staph. aureus often causes subclinical and chronic mastitis and affects all lactating mammals (Contreras and Rodriguez, 2011). Accumulating evidence has shown that Staph. aureus can invade and replicate within cells (Garzoni and Kelley, 2009; Sinha and Fraunholz, 2010), resulting in persistent infection and causing chronic disease.

Autophagy is a crucial degradation mechanism within cells. Cells metabolize nutrients or unnecessary cytoplasmic components through autophagy degradation while maintaining ATP production and macromolecular synthesis. Autophagy is highly conserved in all eukaryotic cells and is regulated by autophagy-related genes (ATG). The ATG12-ATG5 conjugate and microtubule-associated protein 1 light chain 3 (MAP1LC3, also called LC3) are 2 important ubiquitin-like systems participating in autophagy (Ohsumi, 2001). The ATG5 protein is covalently linked to ATG12 and ATG16L1 to form a complex and is conjugated to phagocytic vacuoles and lipidated LC3 (Mizushima et al., 2001). Lipidated LC3 and its homologs or paralogs are involved in membrane elongation and fusion, eventually forming a closed double-membrane vesicle (called an autophagosome), which is the main morphological feature of autophagy (Kabeya et al., 2000). 
Autophagy participates in the elimination of invading pathogenic organisms (Rich et al., 2003). Selective autophagy is mediated through the interaction of the ubiquitinated pathogenic microorganisms with autophagy receptors, including p62 (Johansen and Lamark, 2011), nuclear dot protein 52 (Thurston et al., 2009), and optineurin (Wild et al., 2011). Finally, the autophagosomes engulf the pathogens and fuse with lysosomes to exert a degradative effect. However, some bacteria have evolved strategies to evade such elimination by interfering with autophagy, thus creating favorable conditions for their own survival. Ogawa et al. (2005) reported that Shigella could secrete IcsB protein to escape the autophagy of host cells, thereby causing intracellular infection. Wang et al. (2009) reported that autophagy was induced by Helicobacter pylori in macrophages, but the bacteria could adapt and proliferate in autophagic vesicles. Recently, it has been shown that Staph. aureus modulates the host's central carbon metabolism to activate autophagy and sustain its own intracellular survival in HeLa cells and human umbilical vein endothelial cells (HUVEC; Bravo-Santano et al., 2018).

Whether autophagy is involved in the process of Staph. aureus invading bovine mammary epithelial cells (BMEC) has not been reported yet, and the specific roles of autophagy in the invasive procedure are unclear. The aim of this study was to investigate the expression of autophagy during infection and the effect of autophagosome formation and degradation on the proliferation of intracellular Staph. aureus in BMEC.

\section{MATERIALS AND METHODS}

\section{Reagents}

Fetal bovine serum (FBS) and other tissue culture reagents were purchased from Gibco BRL Co. (Grand Island, NY). Dulbecco's modified Eagle's medium/F12 (DMEM/F12), rapamycin, 3-methyladenine (3-MA), and chloroquine (CQ) were obtained from SigmaAldrich (St. Louis, MO). Anti-LC3 was from Medical \& Biological Laboratories Co. Ltd. (Nagoya, Japan). $\beta$-Actin, p62, and horseradish peroxidase (HRP)-conjugated secondary antibodies were supplied by Cell Signaling Technology (Boston, MA). Lipofectamine 2000 was from Invitrogen Life Technologies (Carlsbad, CA).

\section{Culture of BMEC}

Mammary tissues were collected aseptically from healthy lactating Holstein cows and cleaned with PBS containing $300 \mathrm{IU}$ of penicillin/streptomycin to remove the milk and blood. The cows were from the University of Yangzhou herd, and the protocol was approved by the Animal Care and Ethics Committee of Yangzhou University (approval ID: YZUDWSY2017-0029). Following collection, the samples were transported to the laboratory for further treatment. Mammary tissues were rinsed with PBS, and acini were selected and transferred into another beaker. Then, mammary acini were cut with ophthalmic scissors into paste-like blocks. DMEM/F12 was applied to suspend and wash the blocks until the suspension was clear. Collagenase (EC 3.4.24.3) was added into the acinus blocks and digested at $37^{\circ} \mathrm{C}$ and $120 \mathrm{rpm}$ for 2 to $3 \mathrm{~h}$ in an incubator. Then, 20- and 80-mesh strainers were used successively to filter the digested cell suspension. Cells were collected and resuspended in DMEM/F12 medium containing $15 \%$ FBS and $100 \mathrm{IU}$ of penicillin/streptomycin; these cells were then seeded in cell culture flasks and cultured at $37^{\circ} \mathrm{C}$ with $5 \% \mathrm{CO}_{2}$. When the cells reached $80 \%$ confluency, they were purified according to differing sensitivity to trypsin and identified by immunohistochemistry.

\section{Bacterial Strains and Culture}

Staphylococcus aureus (ATCC 29213), which was recovered and cultured in Luria-Bertani liquid and solid media at $37^{\circ} \mathrm{C}$, was used in this study. Bacterial growth was monitored by measuring the optical density at $600 \mathrm{~nm}\left(\mathrm{OD}_{600}\right)$. When Staph. aureus entered the logarithmic growth phase, the bacteria were used in experiments.

\section{Infection}

Staphylococcus aureus in the logarithmic growth phase was diluted in DMEM/F12 to obtain the desired multiplicity of infection (1:1). Cells were then infected with Staph. aureus for $1.5 \mathrm{~h}$. Subsequently, the cells were treated with $10 \mu \mathrm{g} / \mathrm{mL}$ lysostaphin (EC 3.4.24.75) for 12 min to kill all extracellular bacteria, and we recorded this time as $0 \mathrm{~h}$ post-infection (hpi). Cells starved for $3 \mathrm{~h}$ in Hanks' balanced salt solution (HBSS) served as the autophagy positive group. For the autophagy induction or inhibition experiments, cells were treated with different concentrations of rapamycin, 3-MA, or CQ before being infected with the bacterium.

\section{Western Blot Analysis}

Cells were washed with PBS 3 times and then scraped off the plate. Total protein was extracted, and protein concentrations were detected with a Bicinchoninic Acid 
Protein Assay kit (Beyotime, Shanghai, China). Total proteins were separated by SDS-PAGE and transferred to polyvinyl difluoride membranes (Millipore, Billerica, MA). Nonspecific binding was reduced by placing the membranes in 5\% skim milk for $1 \mathrm{~h}$. Then, the membranes were immunoblotted with primary antibodies specific for $\mathrm{LC} 3, \mathrm{p} 62$, and $\beta$-actin at $4^{\circ} \mathrm{C}$ overnight, and the membranes were washed in Tris-buffered saline/ Tween (TBS-T) buffer and incubated with HRPconjugated secondary antibodies at room temperature for $1 \mathrm{~h}$. After 3 additional washes in TBS-T, the blots were layered with enhanced chemiluminescence (ECL) reagent (Millipore, Billerica, MA) and detected by using a chemiluminescence imaging system (ChemiScope 5300; Clinx Science Instruments, Shanghai, China).

\section{Immunofluorescence Analysis}

The cells were fixed with $4 \%$ paraformaldehyde for 20 min. After being washed with PBS, the cells were incubated with $0.3 \%$ Triton X-100 (Solarbio, Beijing, China) for $15 \mathrm{~min}$ to permeate the membranes and were then blocked with 5\% BSA (Solarbio) for $1 \mathrm{~h}$ at room temperature. Primary antibody incubation of LC3 took place over $1 \mathrm{~h}$ at room temperature. After being washed with PBS, the cells were incubated with goat anti-mouse IgG $(\mathrm{H}+\mathrm{L})$ Alexa Fluor Plus 488 (Thermo Fisher Scientific, Rockford, IL) for $1 \mathrm{~h}$ at room temperature. Then, the cells were stained with 4',6-diamidino-2-phenylindole (DAPI; Beyotime, Shanghai, China). Finally, the coverslips were fixed using fluorescence mounting medium, and the cells were examined by laser scanning confocal microscope (Leica TCS SP8; Leica Corp., Wetzlar, Germany).

\section{Transfection and Fluorescence Colocalization}

The BMEC were plated on coverslips in 24-well cell culture plates. When cells were $80 \%$ confluent, they were transiently transfected with $1 \mu \mathrm{g} / \mathrm{mL}$ green fluorescent protein-LC3 (GFP-LC3) plasmid DNA per well with Lipofectamine 2000 according to the manufacturer's instructions. The medium was replaced after $6 \mathrm{~h}$ with DMEM/F12 containing 15\% FBS. The BMEC were infected with Staph. aureus for $3 \mathrm{~h}$. Then, the cells were collected and fixed with $4 \%$ paraformaldehyde, and the nuclei were labeled with DAPI. LysoTracker Red (Beyotime, Shanghai, China) was added to the culture solution of each well for $1.5 \mathrm{~h}$ before fixation with paraformaldehyde. Finally, the coverslips were fixed using fluorescence mounting medium, and colocalization was assessed using a laser scanning confocal microscope (Leica TCS SP8; Leica Corp.).

\section{Intracellular Survival Assay}

Autophagy in the BMEC was improved by rapamycin and inhibited by CQ and 3-MA. Staphylococcus aureus was added into the cells at a multiplicity of infection of $1: 1$. After $1.5 \mathrm{~h}$, the cells were treated with $10 \mu \mathrm{g} /$ $\mathrm{mL}$ lysostaphin for $12 \mathrm{~min}$ to kill extracellular bacteria. Then, the cells were washed with PBS to wash out extracellular bacteria. The samples were collected at 1 , 2,3 , and $4 \mathrm{hpi}$, respectively, and were lysed by addition of $0.5 \%$ Triton X-100. Serial dilutions were plated on Luria-Bertani agar for bacterial enumeration. Three replications were performed for each group.

\section{Statistical Analysis}

The experimental data were analyzed using SPSS 17.0 software (SPSS Inc./IBM Corp., Chicago, IL) using one-way ANOVA and Dunnett's test. The data were presented as the means \pm standard error of the mean. A $P$-value $<0.05$ between 2 sets of data was considered statistically significant. Each experiment was repeated 3 times.

\section{RESULTS}

\section{Protein Expression of LC3-II and p62 in BMEC Challenged by Staph. aureus}

To determine whether autophagy in BMEC could be induced during Staph. aureus infection, we detected protein expression levels of LC3 and p62 by Western blot. As shown in Figure 1b, protein expression of LC3II in the cells increased $(P<0.01)$ at 2,3 , and $4 \mathrm{hpi}$ compared with the control group. No difference $(P>$ 0.05 ) was observed in LC3-II protein expression at 2,3 , and 4 hpi compared with the HBSS group. The protein expression of p62 increased $(P<0.01)$ at 3 and 4 hpi compared with the control and HBSS groups (Figure 1c).

\section{Observation of LC3 Puncta in BMEC Infected by Staph. aureus}

To further verify the induction of autophagy after infection of BMEC, immunofluorescence analysis was applied to detect LC3 puncta. As shown in Figure 2, more green fluorescence cluster points (LC3 puncta) appeared after Staph. aureus inoculation at $3 \mathrm{hpi}$. In the control group, the fluorescence intensity of the cells was weak, and no obvious fluorescence cluster points were observed. In the HBSS (positive control) group, we observed green fluorescence cluster points similar to those of the infected group. 


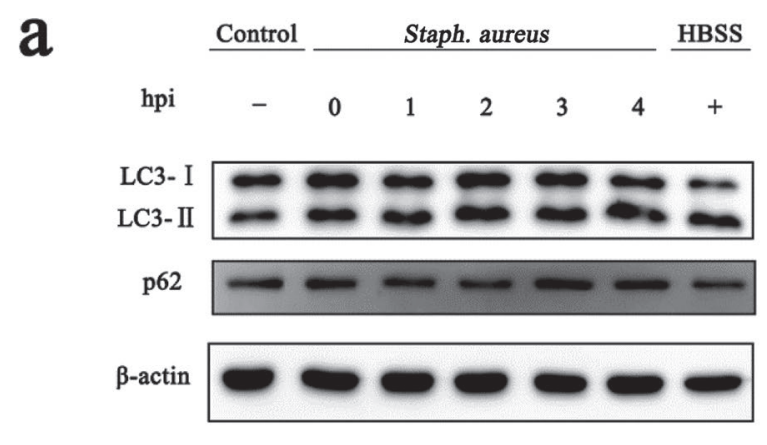

b

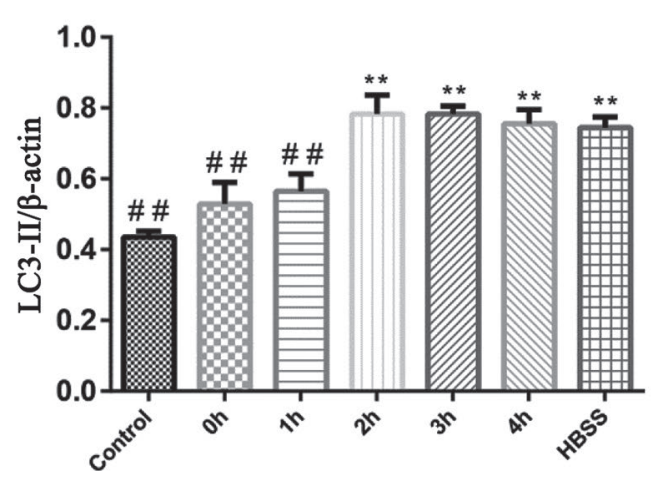

C

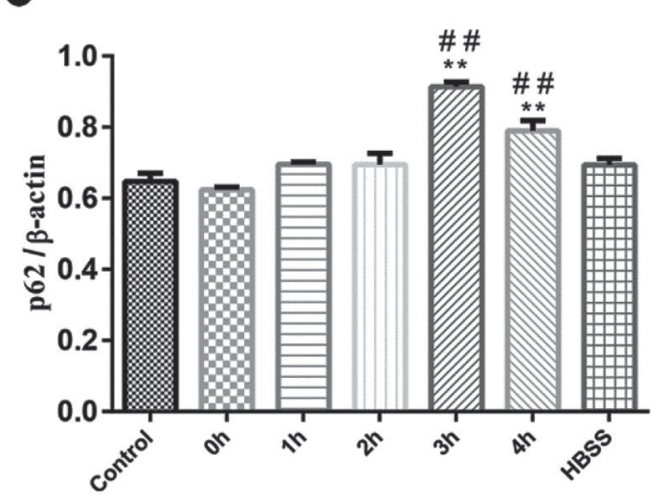

Figure 1. Relative abundance of microtubule-associated protein 1 light chain 3-II (LC3-II) and sequestosome-1 (p62) in bovine mammary epithelial cells (BMEC) after the infection with Staphylococcus aureus. (a) BMEC were infected with Staph. aureus (multiplicity of infection = 1:1) for $1.5 \mathrm{~h}$, and cells were treated with $10 \mu \mathrm{g} / \mathrm{mL}$ of lysostaphin for $12 \mathrm{~min}$ to kill all extracellular bacteria. The cells were collected at $0,1,2$, 3 , and 4 hours post-infection (hpi). Total proteins were prepared, and LC3-II, p62, and $\beta$-actin were examined by Western blot (a). Expression of LC3-II (b) and p62 (c) was measured relative to that of $\beta$-actin. The data are presented as the means \pm SEM. Each experiment was repeated 3 times. ${ }^{* *} P<0.01$ vs. untreated control group; \#\#P<0.01 vs. autophagy positive control group (starved in Hanks' balanced salt solution, HBSS).

\section{Colocalization of Autophagosomes and Lysosomes During Staph. aureus Infection}

The GFP-LC3 plasmid was transfected into BMEC and LysoTracker was applied to mark the lysosomes. As shown in Figure 3, more LC3 green puncta appeared and the lysosomes were marked by LysoTracker as red points in the experimental group. The green puncta did not overlap with the red points. In the control group, the lysosomes could be identified as red points, whereas LC3 green puncta were rarely observed. In the HBSS positive control group, the LC3 green puncta overlapped with the red points.

\section{Effects of Autophagy Inhibitors and Inducers on Protein Expression of LC3 and p62}

To investigate the effects of autophagosome formation and degradation on the protein expression of LC3 and p62 in BMEC under infection, autophagy was regulated with inducer rapamycin and inhibitors 3-MA and CQ; the autophagy inhibitors and inducers were added during infection. As shown in Figure 4b, a significant $(P<$ $0.01)$ increase in LC3-II protein expression occurred in the rapamycin- and CQ-treated groups compared with the control group. In the 3-MA treatment group, the relative abundance of LC3-II was lower $(P<0.05)$ than that of the control group. After co-incubation with rapamycin, CQ, or 3-MA, the expression of p62 protein of these 3 groups increased $(P<0.01)$ compared with the control and HBSS groups (Figure 4c).

\section{Effects of Autophagy Inhibitors and Inducers on Intracellular Proliferation of Staph. aureus During Infection}

To investigate the effects of autophagy on the intracellular proliferation of Staph. aureus, autophagy regulatory reagents were added to BMEC during the infection. The number of intracellular bacteria was determined with an intracellular survival assay. As shown in Figure 5, the number of intracellular bacteria in the 
rapamycin treatment group increased $(P<0.05$ or $P$ $<0.01)$ at 1 to 4 hpi compared with the Staph. aureus group. In the CQ treatment group, the number of intracellular bacteria was significantly higher $(P<0.01)$ than in the Staph. aureus group at all test times. In the 3-MA treatment group, the number of intracellular bacteria decreased significantly $(P<0.01)$ at 2,3 , and 4 hpi compared with those in the Staph. aureus group.

\section{DISCUSSION}

Autophagy can act as a mechanism of innate immunity when cells are infected with pathogens (Mestre and Colombo, 2012). As a key protein of autophagy, LC3-II can be stably present on the inner and outer membranes of autophagosomes until the formation of autolysosomes; LC3-I normally presents in the cytosol and is diffusely distributed. When autophagy occurs, LC3-II binds to phosphatidylethanolamine and localizes in autophagic vacuoles. Therefore, LC3-II can be used to label autophagosomes (Kimura et al., 2009). The amount of LC3-II, the LC3-II:LC3-I ratio, or the LC3II:(LC3-I + LC3-II) ratio is currently used to assess autophagy. Because LC3-II tends to be more sensitive than LC3-I in immunoblotting, comparing the amount of LC3-II among samples is likely to be the more accurate method (Mizushima and Yoshimori, 2007) and is frequently used in autophagy analysis (Tan et al., 2015; Neumann et al., 2016; Chu et al., 2018). Here, we found that the protein flux of LC3-II increased from 2 to 4 hpi, and LC3 puncta appeared at $3 \mathrm{hpi}$, which suggests the induction of autophagy by BMEC during Staph. aureus infection. This observation was consistent with previous studies showing that selective autophagy was induced after invasion of Staph. aureus into the host cells (Neumann et al., 2016). Recent studies found that Staph. aureus severely depleted the glucose and amino acid pools and induced breakdown of glutamine by the host cells to meet its own metabolic needs, which activated autophagy in the cells (Bravo-Santano et al., 2018).

Nucleoporin p62 is an autophagy receptor protein, which can capture the ubiquitinated proteins or pathogens involved in selective autophagy (Noda et al., 2010). It binds to LC3 through the LIR (LC3 interacting region) domain to form a complex, which is degraded as autophagy-specific substrates in autolysosomes (Thurston et al., 2009; Park et al., 2014).
DAPI
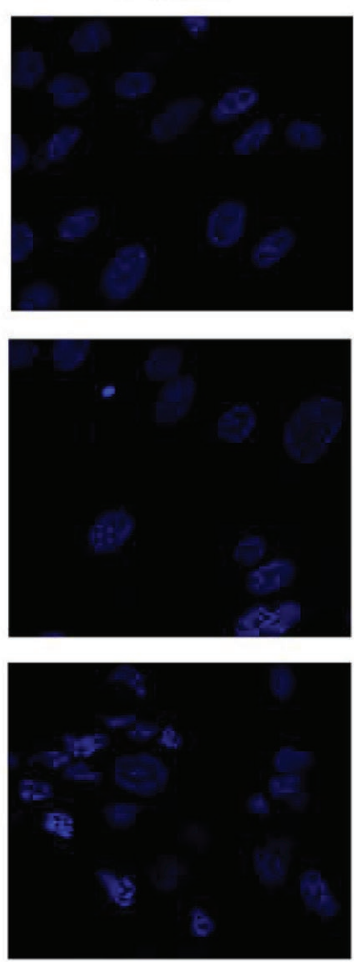

LC3
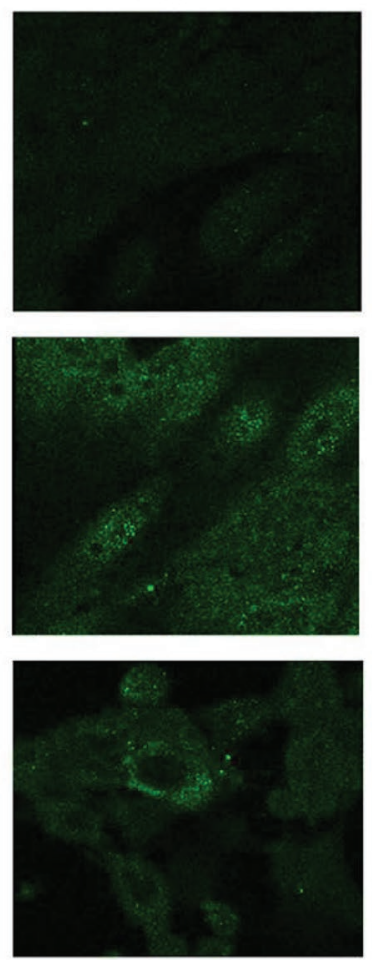

Merge
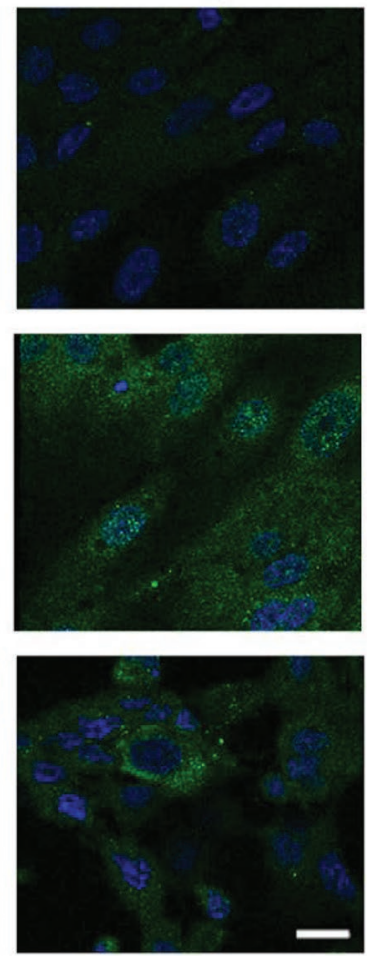

Figure 2. Autophagosome formation in bovine mammary epithelial cells (BMEC) induced by Staphylococcus aureus (multiplicity of infection $=1: 1$ ) for $3 \mathrm{~h}$ post-infection, untreated (control), or starved in Hanks' balanced salt solution (HBSS). Subsequently, the cells were fixed and

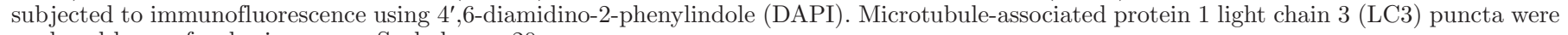
analyzed by confocal microscopy. Scale bar $=20 \mu \mathrm{m}$. 


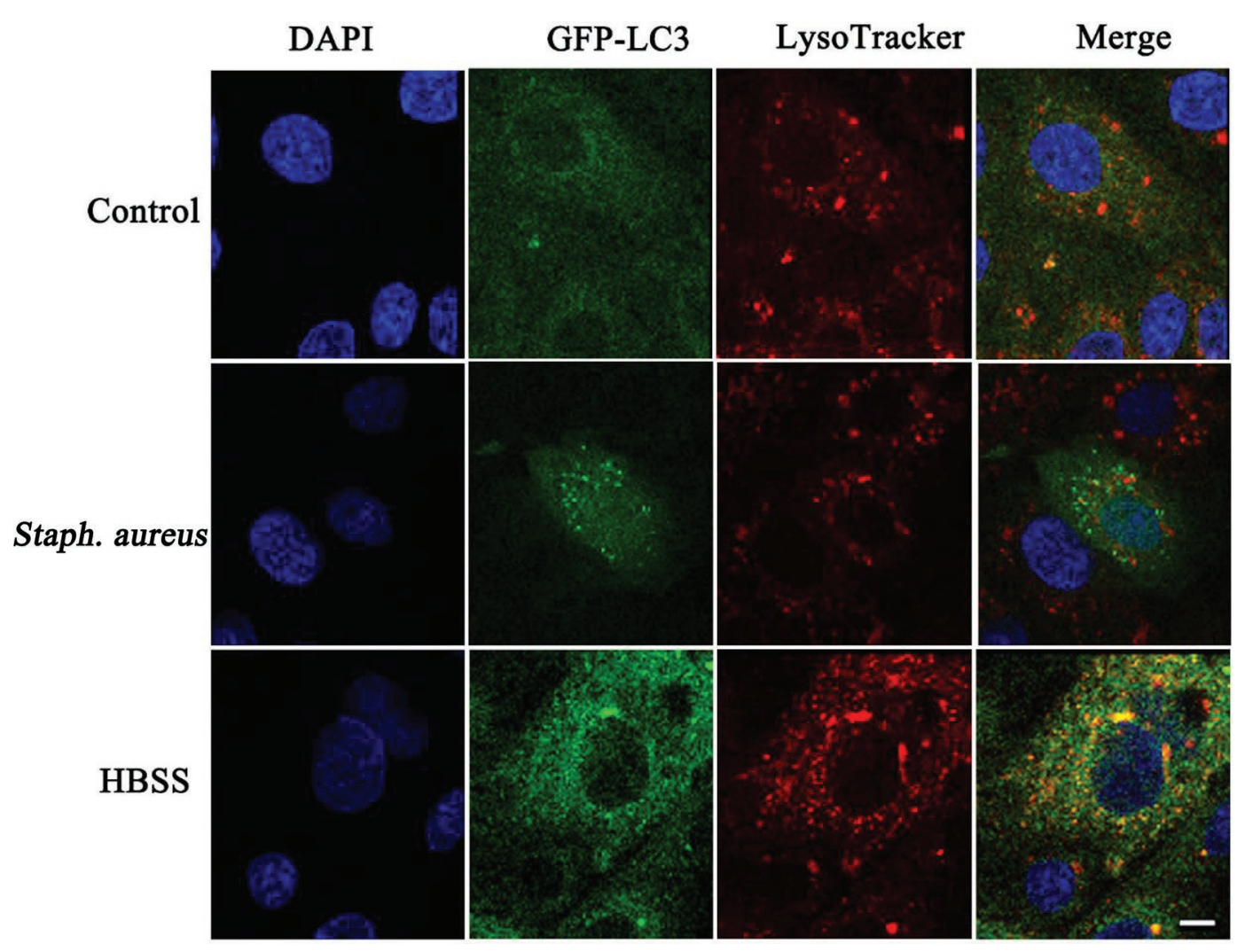

Figure 3. Colocalization of autophagosomes and lysosomes in bovine mammary epithelial cells (BMEC). The BMEC transfected with green fluorescent protein-microtubule-associated protein 1 light chain 3 (GFP-LC3) were either infected with Staphylococcus aureus (multiplicity of infection $=1: 1$ ) for $3 \mathrm{~h}$ post-infection, left untreated, or starved for $3 \mathrm{~h}$ in Hanks' balanced salt solution (HBSS). LysoTracker (Beyotime, Shanghai, China) was added $1.5 \mathrm{~h}$ before the cells were fixed and the nuclei were labeled with 4',6-diamidino-2-phenylindole (DAPI). Then, colocalization of autophagosomes and lysosomes was analyzed by confocal microscopy. Scale bars $=10 \mu \mathrm{m}$.

The outer membrane of an autophagosome fuses with a lysosome to form an autolysosome (Hyttinen et al., 2013). The inner membrane of the autophagosome is degraded by lysosomal enzymes, and their contents are degraded. Therefore, p62 is often used for the detection of autophagic flux (Larsen et al., 2010). In this study, the protein expression of p62 increased at 3 and $4 \mathrm{hpi}$, which suggests that autophagic flux was hindered. The nonoverlapping puncta of the autophagosomes (LC3) and lysosomes indicated that the fusion of the autophagosome and lysosome in BMEC infected with Staph. aureus was inhibited. These findings were consistent with the results of a previous study showing that Staph. aureus containing $\alpha$-hemolysin could induce autophagy in Chinese hamster ovary (CHO) cells, thus preventing the maturation of autophagosomes (Mestre et al., 2010). This result may suggest the possibility of survival for Staph. aureus in the autophagosomes, thereby causing persistent infection.

Autophagy is involved in both the innate and adaptive immune responses, and some microorganisms can be eliminated by the autophagic mechanism (Levine et al., 2011; Deretic et al., 2013). The evasion of autophagic elimination by bacteria has been documented (Castrejón-Jiménez et al., 2015). Porphyromonas gingivalis, a pathogen that causes periodontitis, can delay the maturation of autophagosomes and inhibit the production of autolysosomes (Dorn et al., 2001). Therefore, it can avoid lysosomal degradation to colonize in autophagosomes and replicate. Listeria monocytogenes, the only strain of Listeria that causes human disease, can utilize a cholesterol-dependent pore-forming toxin, listeriolysin $\mathrm{O}$, to escape degradation by autophagy (Birmingham et al., 2008). Salmonella can suppress autophagy via an Akt/mTOR signaling pathway by activation of focal adhesion kinase (Owen et al., 2014). In this study, we applied autophagy inhibitors and inducers to regulate autophagy and observed the function of autophagy in the survival of Staph. aureus in BMEC. The level of autophagy was elevated after Staph. aureus infection of BMEC. When BMEC were treated with rapamycin, the formation of autophagic vacuoles increased and protein expression of $\mathrm{p} 62$ was enhanced, which indicated that the increase in autophagy level did not promote the 


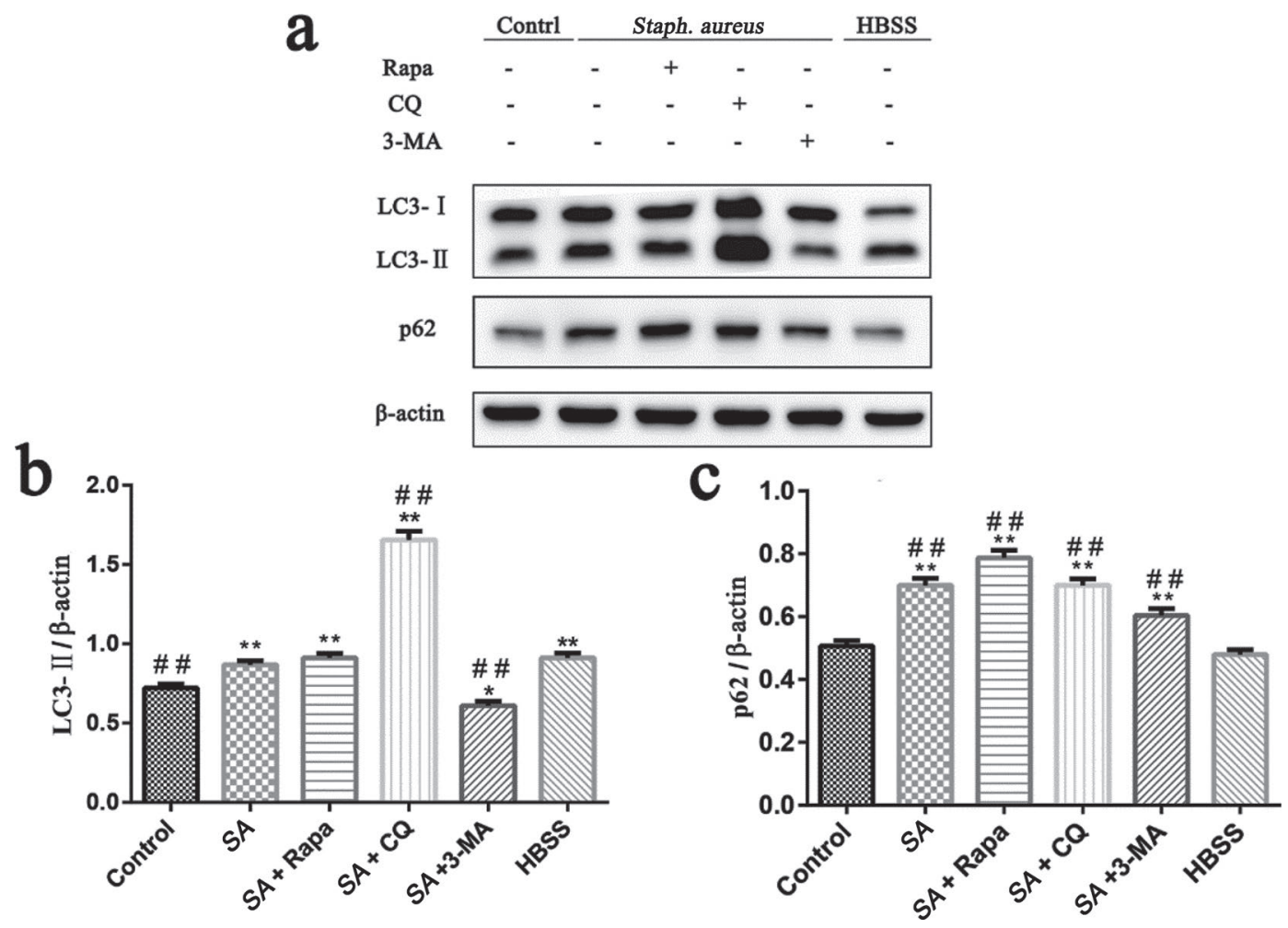

Figure 4. The protein expression of microtubule-associated protein 1 light chain 3-II (LC3-II) and sequestosome-1 (p62) in bovine mammary epithelial cells (BMEC) after infection with Staphylococcus aureus (SA) and treatment with autophagy inhibitors and inducers. (a) The BMEC were infected with Staph. aureus (multiplicity of infection = 1:1) for 3 h post-infection. Rapamycin (Rapa), 3-methyladenine (3-MA), or chloroquine $(\mathrm{CQ})$ was added in each experimental group during the infection. Total proteins were prepared, and LC3-II, p62, and $\beta$-actin were examined by Western blot (a). Expression of LC3-II (b) and p62 (c) was measured relative to that of $\beta$-actin. The data are presented as the means \pm SEM. Each experiment was repeated 3 times. ${ }^{*} P<0.05$ vs. control group; ${ }^{* *} P<0.01$ vs. control group; \#\#P<0.01 vs. autophagy positive control group (starved in Hanks' balanced salt solution, HBSS).

degradation of autolysosomes. Meanwhile, the bacterial load in BMEC also increased, which confirmed that autophagy induction could not contribute to clearance of the bacteria. In contrast, the accumulated autophagosomes may provide a niche for the intracellular replication of Staph. aureus. These data verified that an autophagy imbalance could contribute to the survival or replication of Staph. aureus in BMEC, thus partly explaining persistent Staph. aureus infection in dairy mastitis. Schnaith et al. (2007) also found that more Staph. aureus were recovered in HeLa cells treated with rapamycin. The protein expression of LC3-II in BMEC was improved with $\mathrm{CQ}$, but the expression of p62 also increased, and more Staph. aureus were recovered from the intracellular compartments of BMEC, which may be due to lysosome alkalinization induced by CQ. The degradation of autolysosomes was prevented. A greater number of autophagosomes facilitated Staph. aureus survival in BMEC. These findings are similar to previous results that showed bacteria could replicate in autophagosomes and eventually cause persistent infection when the maturation and degradation of host autopha- gosomes were delayed or inhibited (Dorn et al., 2001; Birmingham et al., 2008). The formation of autophagosomes could be inhibited by 3-MA, and the intracellular bacterial load was decreased compared with the control group, which indicated that the decrease in the number of autophagosomes could reduce the bacterial load of Staph. aureus in BMEC. Previous studies have shown that inhibition of the autophagic pathway by treatment with 3-MA could restore intracellular bactericidal action (Schnaith et al., 2007; O'Keeffe et al., 2015), which was similar to our results. Therefore, we concluded that formation of autophagosomes facilitated the replication of Staph. aureus in BMEC.

\section{CONCLUSIONS}

Staphylococcus aureus infection induced autophagy in BMEC but autophagosomes and lysosomes could not fuse properly and autophagic flux was obstructed. Staphylococcus aureus allows the undegradable autophagosome to proliferate. Inhibiting the formation of autophagosomes in BMEC facilitated the clearance of 


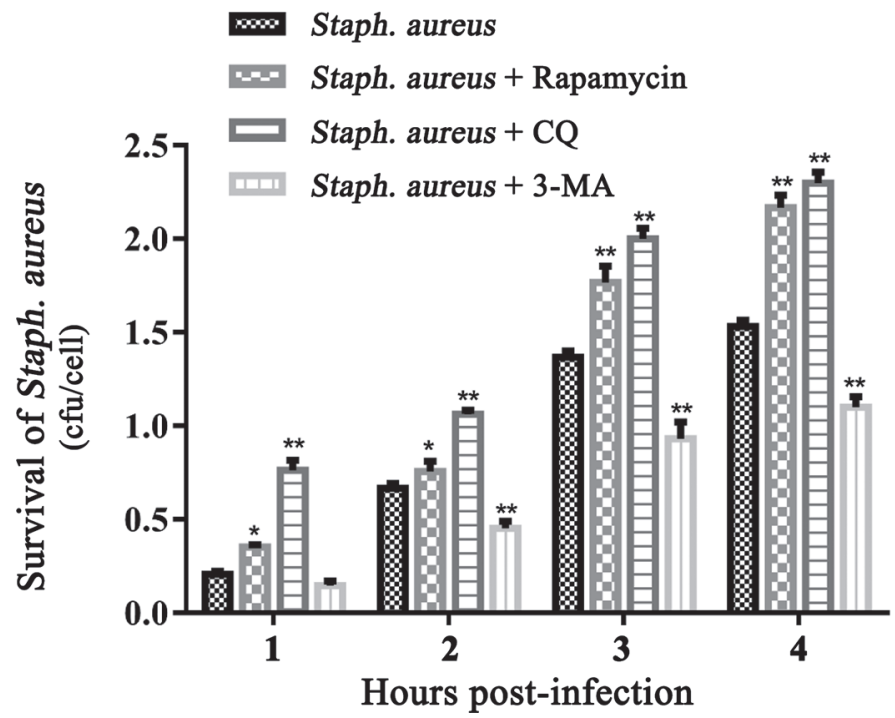

Figure 5. The effect of autophagy on intracellular survival of Staphylococcus aureus in bovine mammary epithelial cells (BMEC). The BMEC were infected with Staph. aureus (multiplicity of infection $=1: 1$ ) and treated with rapamycin, 3-methyladenine (3-MA), or chloroquine (CQ). Survival of intracellular Staph. aureus was measured at $1,2,3$, and $4 \mathrm{~h}$ post-infection. The data are presented as the means \pm SEM. Each experiment was repeated 3 times. ${ }^{*} P<0.05$ and ${ }^{* *} P<$ 0.01 vs. Staph. aureus group.

intracellular Staph. aureus, and this may offer a new strategy to treat mastitis in cows.

\section{ACKNOWLEDGMENTS}

The investigation was supported by the Natural Science Foundation of Jiangsu Province (Nanjing, China; grant no. BK20160062), National Natural Science Foundation of China (Beijing, China; grant no. 31302151), earmarked fund for Jiangsu Agricultural Industry Technology System [Nanjing, China; grant no. JATS(2018)315], Outstanding Young Backbone Teacher Foundation of Yangzhou University Priority Academic Program Development of Jiangsu Higher Education Institutions (PAPD; Nanjing, China), and Top-notch Academic Programs Project of Jiangsu Higher Education Institutions (TAPP; Nanjing, China).

\section{REFERENCES}

Birmingham, C. L., D. E. Higgins, and J. H. Brumell. 2008. Avoiding death by autophagy: Interactions of Listeria monocytogenes with the macrophage autophagy system. Autophagy 4:368-371.

Bravo-Santano, N., J. K. Ellis, L. M. Mateos, Y. Calle, H. C. Keun, V. Behrends, and M. Letek. 2018. Intracellular Staphylococcus aureus modulates host central carbon metabolism to activate autophagy. mSphere 3:e00374-18.

Castrejón-Jiménez, N. S., K. Leyva-Paredes, J. C. Hernandez-Gonzalez, J. Luna-Herrera, and B. E. Garcia-Perez. 2015. The role of autophagy in bacterial infections. Biosci. Trends 9:149-159.
Chu, B. X., R. F. Fan, S. Q. Lin, D. B. Yang, Z. Y. Wang, and L. Wang. 2018. Interplay between autophagy and apoptosis in lead(II)-induced cytotoxicity of primary rat proximal tubular cells. J. Inorg. Biochem. 182:184-193.

Contreras, G. A., and J. M. Rodriguez. 2011. Mastitis: Comparative etiology and epidemiology. J. Mammary Gland Biol. Neoplasia 16:339-356.

Deretic, V., T. Saitoh, and S. Akira. 2013. Autophagy in infection, inflammation and immunity. Nat. Rev. Immunol. 13:722-737.

Dorn, B. R., W. A. Dunn Jr., and A. Progulske-Fox. 2001. Porphyromonas gingivalis traffics to autophagosomes in human coronary artery endothelial cells. Infect. Immun. 69:5698-5708.

Garzoni, C., and W. L. Kelley. 2009. Staphylococcus aureus: New evidence for intracellular persistence. Trends Microbiol. 17:59-65.

Halasa, T., K. Huijps, O. Osteras, and H. Hogeveen. 2007. Economic effects of bovine mastitis and mastitis management: A review. Vet. Q. 29:18-31.

Hillerton, J. E., and E. A. Berry. 2005. Treating mastitis in the cowA tradition or an archaism. J. Appl. Microbiol. 98:1250-1255.

Hyttinen, J. M. T., M. Niittykoski, A. Salminen, and K. Kaarniranta. 2013. Maturation of autophagosomes and endosomes: A key role for Rab7. Biochim. Biophys. Acta Mol. Cell Res. 1833:503-510.

Johansen, T., and T. Lamark. 2011. Selective autophagy mediated by autophagic adapter proteins. Autophagy 7:279-296.

Joshi, S., and S. Gokhale. 2006. Status of mastitis as an emerging disease in improved and periurban dairy farms in India. Ann. N. Y. Acad. Sci. 1081:74-83.

Kabeya, Y., N. Mizushima, T. Uero, A. Yamamoto, T. Kirisako, T. Noda, E. Kominami, Y. Ohsumi, and T. Yoshimori. 2000. LC3, a mammalian homologue of yeast Apg8p, is localized in autophagosome membranes after processing. EMBO J. 19:5720-5728.

Kimura, S., N. Fujita, T. Noda, and T. Yoshimori. 2009. Monitoring autophagy in mammalian cultured cells through the dynamics of Lc3. Methods Enzymol. 452:1-12.

Larsen, K. B., T. Lamark, A. Overvatn, I. Harneshaug, T. Johansen, and G. Bjorkoy. 2010. A reporter cell system to monitor autophagy based on p62/SQSTM1. Autophagy 6:784-793.

Levine, B., N. Mizushima, and H. W. Virgin. 2011. Autophagy in immunity and inflammation. Nature 469:323-335.

Mestre, M. B., and M. Colombo. 2012. Staphylococcus aureus promotes autophagy by decreasing intracellular cAMP levels. Autophagy $8: 1865-1867$.

Mestre, M. B., C. M. Fader, C. Sola, and M. I. Colombo. 2010. Alphahemolysin is required for the activation of the autophagic pathway in Staphylococcus aureus-infected cells. Autophagy 6:110-125.

Mizushima, N., A. Yamamoto, M. Hatano, Y. Kobayashi, Y. Kabeya, K. Suzuki, T. Tokuhisa, Y. Ohsumi, and T. Yoshimori. 2001. Dissection of autophagosome formation using Apg5-deficient mouse embryonic stem cells. J. Cell Biol. 152:657-668.

Mizushima, N., and T. Yoshimori. 2007. How to interpret LC3 immunoblotting. Autophagy 3:542-545.

Neumann, Y., S. A. Bruns, M. Rohde, T. K. Prajsnar, S. J. Foster, and I. Schmitz. 2016. Intracellular Staphylococcus aureus eludes selective autophagy by activating a host cell kinase. Autophagy 12:2069-2084.

Noda, N. N., Y. Ohsumi, and F. Inagaki. 2010. Atg8-family interacting motif crucial for selective autophagy. FEBS Lett. 584:1379-1385.

O'Keeffe, K. M., M. M. Wilk, J. M. Leech, A. G. Murphy, M. Laabei, I. R. Monk, R. C. Massey, J. A. Lindsay, T. J. Foster, J. A. Geoghegan, and R. M. McLoughlin. 2015. Manipulation of autophagy in phagocytes facilitates Staphylococcus aureus bloodstream infection. Infect. Immun. 83:3445-3457.

Ogawa, M., T. Yoshimori, T. Suzuki, H. Sagara, N. Mizushima, and C. Sasakawa. 2005. Escape of intracellular Shigella from autophagy. Science 307:727-731.

Ohsumi, Y. 2001. Molecular dissection of autophagy: Two ubiquitinlike systems. Nat. Rev. Mol. Cell Biol. 2:211-216.

Olivares-Pérez, J., A. E. Kholif, S. Rojas-Hernandez, M. M. M. Y. Elghandour, A. Z. M. Salem, A. Z. Bastida, D. Velazquez-Reynoso, M. Cipriano-Salazar, L. M. Camacho-Diaz, M. U. Alonso-Fresan, and N. DiLorenzo. 2015. Prevalence of bovine subclinical mastitis, 
its etiology and diagnosis of antibiotic resistance of dairy farms in four municipalities of a tropical region of Mexico. Trop. Anim. Health Prod. 47:1497-1504.

Owen, K. A., C. B. Meyer, A. H. Bouton, and J. E. Casanova. 2014 Activation of focal adhesion kinase by Salmonella suppresses autophagy via an Akt/mTOR signaling pathway and promotes bacterial survival in macrophages. PLoS Pathog. 10:e1004159.

Park, S., S. G. Choi, S. M. Yoo, J. H. Son, and Y. K. Jung. 2014. Choline dehydrogenase interacts with SQSTM1/p62 to recruit LC3 and stimulate mitophagy. Autophagy 10:1906-1920.

Pitkälä, A., M. Haveri, S. Pyorala, V. Myllys, and T. Honkanen-Buzalski. 2004. Bovine mastitis in Finland 2001-Prevalence, distribution of bacteria, and antimicrobial resistance. J. Dairy Sci. 87:2433-2441.

Rich, K. A., C. Burkett, and P. Webster. 2003. Cytoplasmic bacteria can be targets for autophagy. Cell. Microbiol. 5:455-468.

Schnaith, A., H. Kashkar, S. A. Leggio, K. Addicks, M. Kronke, and O. Krut. 2007. Staphylococcus aureus subvert autophagy for induction of caspase-independent host cell death. J. Biol. Chem. 282:2695-2706.

Sinha, B., and M. Fraunholz. 2010. Staphylococcus aureus host cell invasion and post-invasion events. Int. J. Med. Microbiol. 300:170 175
Tan, X., N. Thapa, Y. Sun, and R. A. Anderson. 2015. A kinaseindependent role for EGF receptor in autophagy initiation. Cell 160:145-160.

Thurston, T. L. M., G. Ryzhakov, S. Bloor, N. von Muhlinen, and F. Randow. 2009. The TBK1 adaptor and autophagy receptor NDP52 restricts the proliferation of ubiquitin-coated bacteria. Nat. Immunol. 10:1215-1221.

Wang, Y. H., J. J. Wu, and H. Y. Lei. 2009. The autophagic induction in Helicobacter pylori-infected macrophage. Exp. Biol. Med. (Maywood) 234:171-180.

Watts, J. L. 1988. Etiological agents of bovine mastitis. Vet. Microbiol. 16:41-66.

Wild, P., H. Farhan, D. G. McEwan, S. Wagner, V. V. Rogov, N. R. Brady, B. Richter, J. Korac, O. Waidmann, C. Choudhary, V. Dotsch, D. Bumann, and I. Dikic. 2011. Phosphorylation of the autophagy receptor optineurin restricts Salmonella growth. Science 333:228-233.

Zhao, X., and P. Lacasse. 2008. Mammary tissue damage during bovine mastitis: Causes and control. J. Anim. Sci. 86(13 Suppl.):57-65. 